\title{
Origin and fate of the nucleolar channel system of normal human endometrium ${ }^{1}$
}

\author{
WANG TzunEng ${ }^{* 2}$ AND J SCHNEIDER ** \\ * Department of Gynecology and Obstetrics, Medical College \\ of Jinan University, Guangzhou, China \\ ** Frauenklinik der Medizinischen Hochschule, Hannover, \\ Germany
}

\begin{abstract}
Human normal endometrium was examined in ultrathin sections. Nucleolar channel system (NCS) appeared in the endometrial epithelial cells during the early and mid secretory phase of menstrual cycle. The NCS was a hollow ball like structure of different sizes and was composed of 2 to 5 rows of tubules embedded in an amorphous matrix. On its surface there were numerous electron dense particles resembling ribosomes. It was usually located within or associated with the nucleolus. Sometimes, it was close to the nuclear envelope or protruding out from the nucleus. On occasion, NCS with simplified structure was found in the perinuclear cytoplasm. Concepts concerning the genesis, involution and function(s) of the NCS were discussed.
\end{abstract}

Key words: nucleolar channel system, human endometrial epithelium, secretory phase.

\section{INTRODUCTION}

Nucleolar channel system(NCS) is usually found in the epithelium of human endometrium during the secretory phase of menstrual cycle $[1,2,3,4,5]$, occasionally also in other tissue cells [6,7]. Theories concerning the origin of NCS remain controversial. Clyman [1] suggested that NCS develops in the nucleolus and subsequently extends toward the nuclear envelope. More et al. [5,8], on the other hand, assumed that NCS is a spiral conformation of tubules originating as an invagination of the inner nuclear membrane. Opinions about the fate of the involuted NCS are also divergent, expulsion from the nucleus [2,3] and intanuclear resorption [2,5] have been proposed. In this study, we reported some observations about the morphology and the location of the NCS, that

1 Supported by a grant from the Volkswagen Stiftung Hannover, Germany

2 Corresponding author. 
Origin and fate of the nucleolar channel system

might contribute to clarify the present ambigous concepts concerning the genesis, involution and function(s) of this system.

\section{MATERIALS AND METHODS}

Endometrial tissue was obtained from corpus uteri of women of reproductive age with normal menstrual cycle, upon whom hysterectomy was performed for benign gynecological diseases not involving the endometrium. The dating was made according to Noyes et al. [9]. One part of this tissue was fixed in formalin solution (10\%) and processed by routine techniques for histological study. The remaining part was processed for electron microscopy by fixing in glutaraldehyde $(2.5 \%)$ dissolved in a 0 . $1 \mathrm{M}$ phosphate - buffered solution and postfixed in osmiumtetroxide (1\%) dissolved in the same buffer. The specimens were then dehydrated in graded alcohols and embedded in Epon 812, sectioned in a Reichert Ultracut ultramicrotome, stained with uranyl acetate and lead citrate, and examined in a JOEL 100 CX II electron microscope.

\section{RESULTS}

NCS was usually found in the endometrial epithelium during early secretory phase (specimen dating 16th, 18th and 19th of the cycle). Occasionally, it could also be found during mid-secretory phase (dating 21 st and 22nd). No NCS could be identified in specimens during proliferative phase and late secretory phase. Morphologically, NCS appeared as a hollow ball-like structure of different sizes. It was composed of 2-5 rows of tubules, encircling a central core containing fine fibrils and granules similar to those of the nucleoplasm (Fig 1,2,3,4). The tubules (ca. 60-90 nm in diameter)were bounded by a unit membrane and filled with fine fibrils. Some amorphous matrix of moderate electron density was found between the tubules. As a rule, NCS was surrounded by heaps of granules with size (ca. $15 \mathrm{~nm}$ in diameter) and electron density like those of the ribosomes covering the rough endoplasmic reticulum ( $\mathrm{rER}$ ) (Fig 1,2,3, 4,7). At times, a piece of the tubule protruding from the NCS into the nucleoplasm could be identified (Fig 4). A direct connection between the tubules of NCS and the perinuclear space could clearly be seen in appropriate sections (Fig 5). NCS was usually embedded in or associated with the nucleolus. In general, there was one set of NCS in a nucleolus, yet 2 appearing within one nucleolus were not too rare (Fig 4,7). Sometimes, NCS laid close to the inner nuclear membrane (Fig 2,3,5) or protruded out from the nucleus (Fig 6). On occasion,NCS without surrounding nucleolar components and directly encircled by a double-layered membrane bearing ribosomes on its outer surface, could be found in the perinuclear cytoplasm(Fig 8).

\section{DISCUSSION}

Morphologically, the contents of the central core of NCS are similar to those forming the nucleoplasm. From our own observations, that the tubules of NCS are in con-tinuity with the perinuclear space (see ref. 4), and that a tubule (connecting tubule?) protrudes from the NCS into the nucleoplasm, we could reasonably assume that the for 
Wang ZN and J Schneider

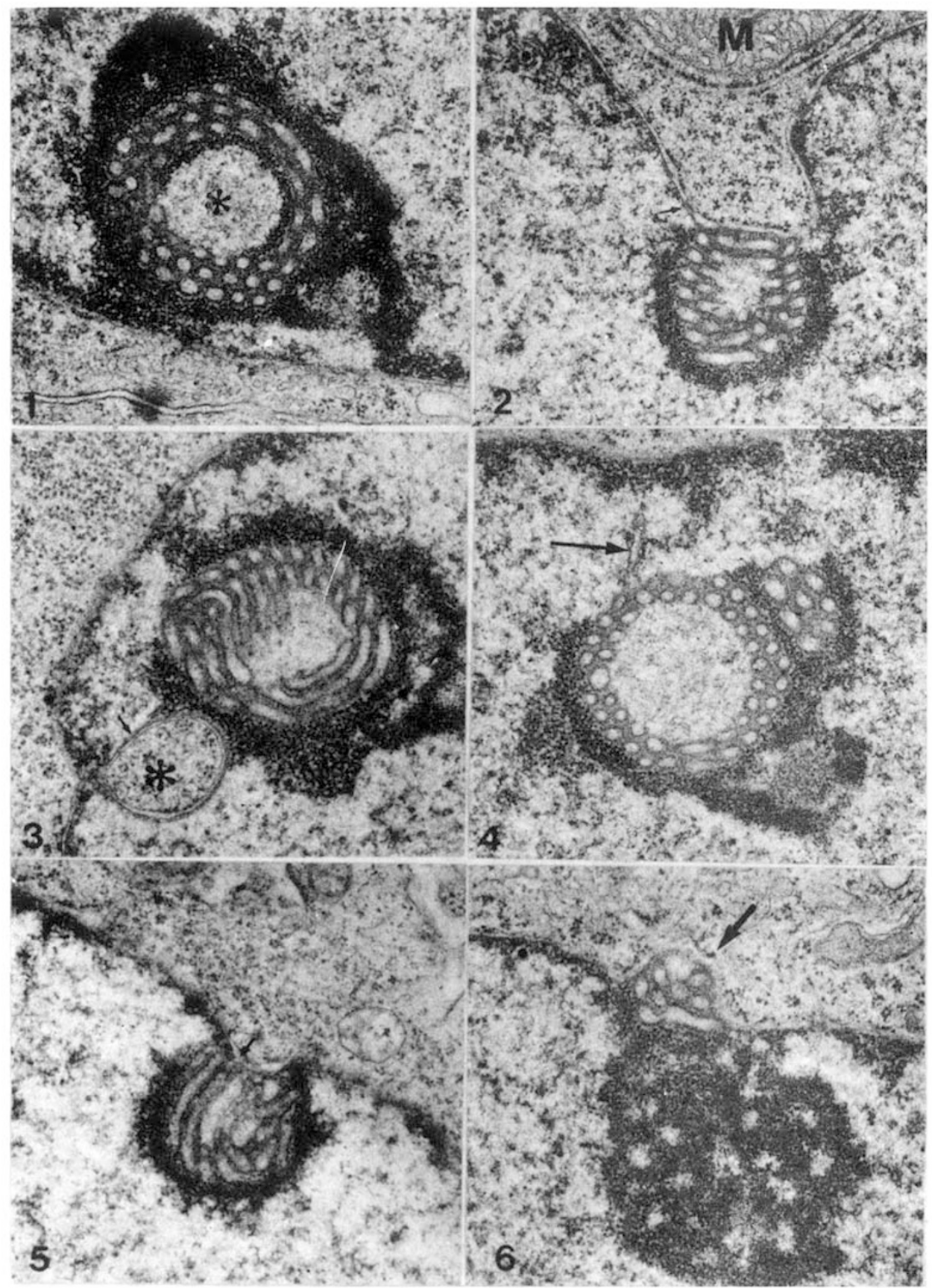

Fig 1. NCS appearing as a hollow ball - like structure. In its central core (*) there are fine fibrils and granules resembling those forming the nucleoplasm. Heaps of $15 \mathrm{~nm}$ granules are seen on the surface of NCS. $\times 27,500$

Fig 2. NCS close to the inner nuclear membrane. Heaps of $15 \mathrm{~nm}$ granules surrounding the NCS, Arrow points to the fibrous lamina associated with the inner nuclear membrane. M: mitochondrion. $\times 27,500$

Fig 3. NCS colse to the inner nuclear membrane. A fibrous lamina (arrow), but no heterochromatin is found on the inner nuclear membrane encircling the cytoplasmic invagination $(*) . \times 27,500$

Fig 4. Two NCS within a nucleolus. Arrow points to a piece of tubule protruding from a NCS into the nucleoplasm. $\times 27,500$

Fig 5. Arrow points to the site of a direct connection between the tubules of NCS and the perinuclear space. $\times 24,000$

Fig 6. NCS protruding from the nuclous (arrow). $\times 27,500$ 
Origin and fate of the nucleolar channel system
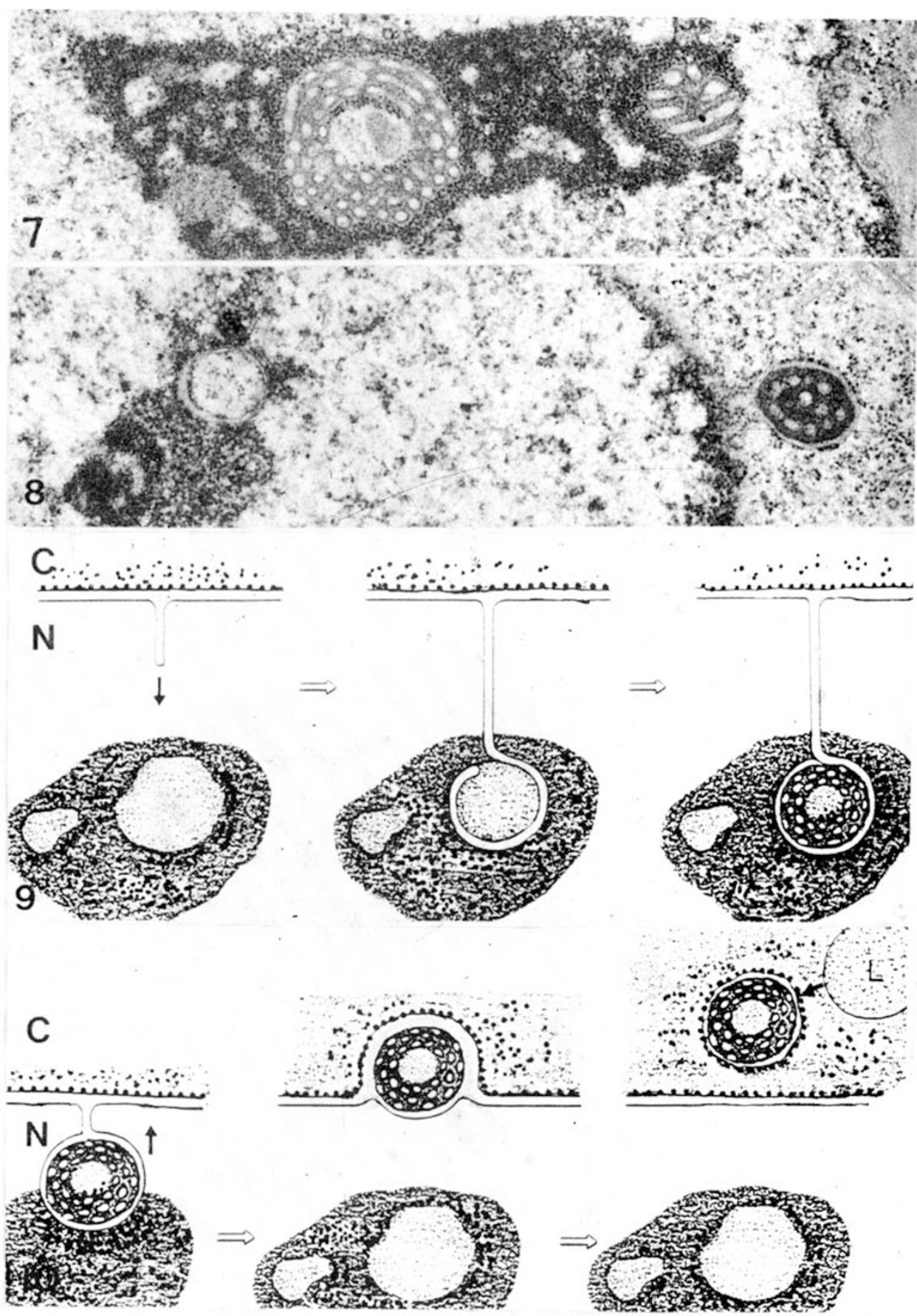

Fig 7. Two NCS appearing in a nucleolus. $\times 25,000$

Fig 8. NCS encircled by a nuclear envelope -like strcture is seen in the perinuclear cytoplasm. $\times 25,000$

Fig 9. Schematic drawing ielustrating the genesis of the NCS. An invagination of the inner nuclear membrane stretching towards the nucleolus, where it coils into multilayers. C: cytoplasm N: nucleus

Fig 10. Schematic drawing ielustrating the involution of the NCS. After being dissociated from the nucleolus, the NCS appears a bud beneath the nuclear envelope. Then it can be discharged into the cytoplasm and disappears following the lysosomal digestion. C: cytoplasm N: nucleus L: lysosome 
mation of NCS is a result of tubular invagination of the inner nuclear membrane into thenucleolus, where it coils spirally into successive layers forming a hollow ball-like structure (Fig 9).

Considering the NCS as an invagination of the inner nuclear membrane, More et al. [5] suggested that the involution of NCS might be a process of intranuclear regression, e.g. re-incorporation of the NCS components with the nuclear membrane. In our study, we found the NCS was protruded from the outer surface of nucleus and was encircled with a nuclear envelope-like structure in the perinuclear cytoplasm. More et al. [5] also found NCS-like structures in lysomes. In respect to involution, all these findings strongly indicated that this system is first dissociated from the nucleolus and appears as bud approching toward the nuclear envelope, then it is discharged from the nucleus into the cytoplasm, where it disappears upon lysosomal digestion(Fig 10). In serial section studies, More and McSeveney [8] observed that NCS was in contact with the nucleolus only in a small area or even not associated with the nucleolus. This might be explained by the possibility that the section planes passed through only a part of the NCS, which was unconnected with the nucleolus.

Although it has been considered that NCS might be a feed-back system between cytoplasm and nucleus for regulating the nucleolar RNA-synthesis or a site for producing specific RNA and/or protein [3]. The concept that it functions as a specialized system for rapid transport of newly synthesized mRNA has been widely accepted [2,3]. However, since the tubules of NCS are open to the perinuclear space, which is known as a prolongation of rER-cisternae, it remains doubtful, whether or not the mRNA molecules could pass through the membrane of rER in order to reach the ribosomes covering the rER. Histochemical study has clearly shown that the electron dense granules surrounding NCS do contain RNA and proteins [3]. Since the occurrence of NCS coincides temporally with the peak of secretory activity of endometrial glands, we prefer to suggest the thesis that NCS might have similar functions as rER, namely the synthesis and transportation of proteins for secretion. The significance of this device is to reduce the burden of rER during the climax of secretion. Alternatively, it might represent a device to increase the surface of the nuclear envelope close to the nucleolus, since the cell needs a large quantity of rRNA during the peak of secretion, and cytoplasmic proteins required to generate ribosomal particles could reach the nucleolus via the NCS.

\section{REFERENCES}

[1] Clyman MJ. A new structure observed in the nucleolus of the human endometrial epithelial cell. Am J Obstet Gynecol 1963; 86:430-32.

[ 2 ] Cornillie FJ, Lauweryns JM. Brosens IA. Normal human endometrium. An ultrastructural survey. Gynecol Obstet Invest 1985; 20:112-29.

[ 3 ] Gordon M. Cyclic changes in the fine structure of the epithelial cells of human endometrium. Int Rev Cytol $1975 ; 42: 127-72$.

[ 4 ] Terzakis JA. The nucleolar channel system of human endometrium. J Cell Biol 1965;27:293-304.

[5] More IA, Armstrong EM, McSeveney D, Chatfield WR. The morphogenesis and fate of the nucleolar channel system in the human endometrial glandular cell. J Ultrastruct Res 1974;44:74-85.

[ 6 ] Daxnerova Z, Mitro A. The nucleolar channel system of nerve cell. Short communication. J Hirnforsch 1984; 25:309-11.

[ 7] Bourgeois CA, Hubert J. Spatial relationship between the nucleolus and the nuclear envelope: Structural as- 
Origin and fate of the nucleolar channel system

pects and functional significance. Int Rev Cytol 1988; 111:1-52.

[ 8] More IA, McSeveney D. The three dimensional structure of the nucleolar channel system in the endometrial glandular cell: Serial sectioning and high voltage electron microscopic studies. J Anat 1980; 130: 673-82.

[ 9 ] Noyes RW, Hertig AT, Rock J. Dating the endometrial biopsy. Fertil Steril 1950; 1:3-25.

Received 15-4-1991. Revised 29-6-1991. Accepted 14-8-1991. 